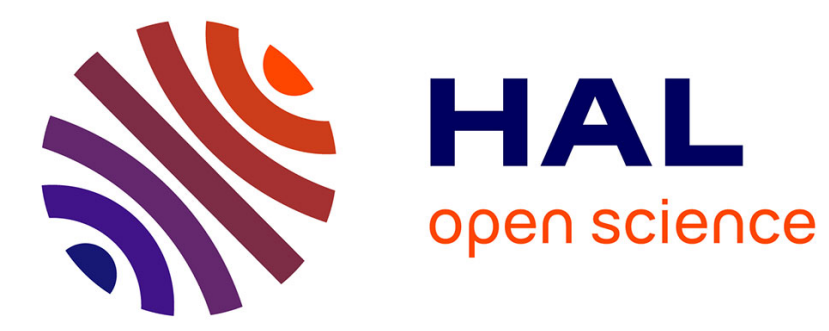

\title{
Un éléphant volant est-il un oiseau? Perspectives pour l'observation hydrométéorologique des milieux de montagne
}

Georges-Marie Saulnier, Thierry Barth

\section{- To cite this version:}

Georges-Marie Saulnier, Thierry Barth. Un éléphant volant est-il un oiseau? Perspectives pour l'observation hydrométéorologique des milieux de montagne. Neige et glace de montagne: Reconstitution, dynamique, pratiques, COLLECTION EDYTEM - Cahiers de Géographie, pp.147-156, 2009. halsde-00404040

\author{
HAL Id: halsde-00404040 \\ https://hal.science/halsde-00404040
}

Submitted on 31 Mar 2011

HAL is a multi-disciplinary open access archive for the deposit and dissemination of scientific research documents, whether they are published or not. The documents may come from teaching and research institutions in France or abroad, or from public or private research centers.
L'archive ouverte pluridisciplinaire HAL, est destinée au dépôt et à la diffusion de documents scientifiques de niveau recherche, publiés ou non, émanant des établissements d'enseignement et de recherche français ou étrangers, des laboratoires publics ou privés. 


\section{Collection EDYTEM}

Numéro 8 - Année 2009

Cahiers de

Géographie

\section{NEIGE et GLACE de MONTAGNE}

Reconstitution, dynamique, pratiques

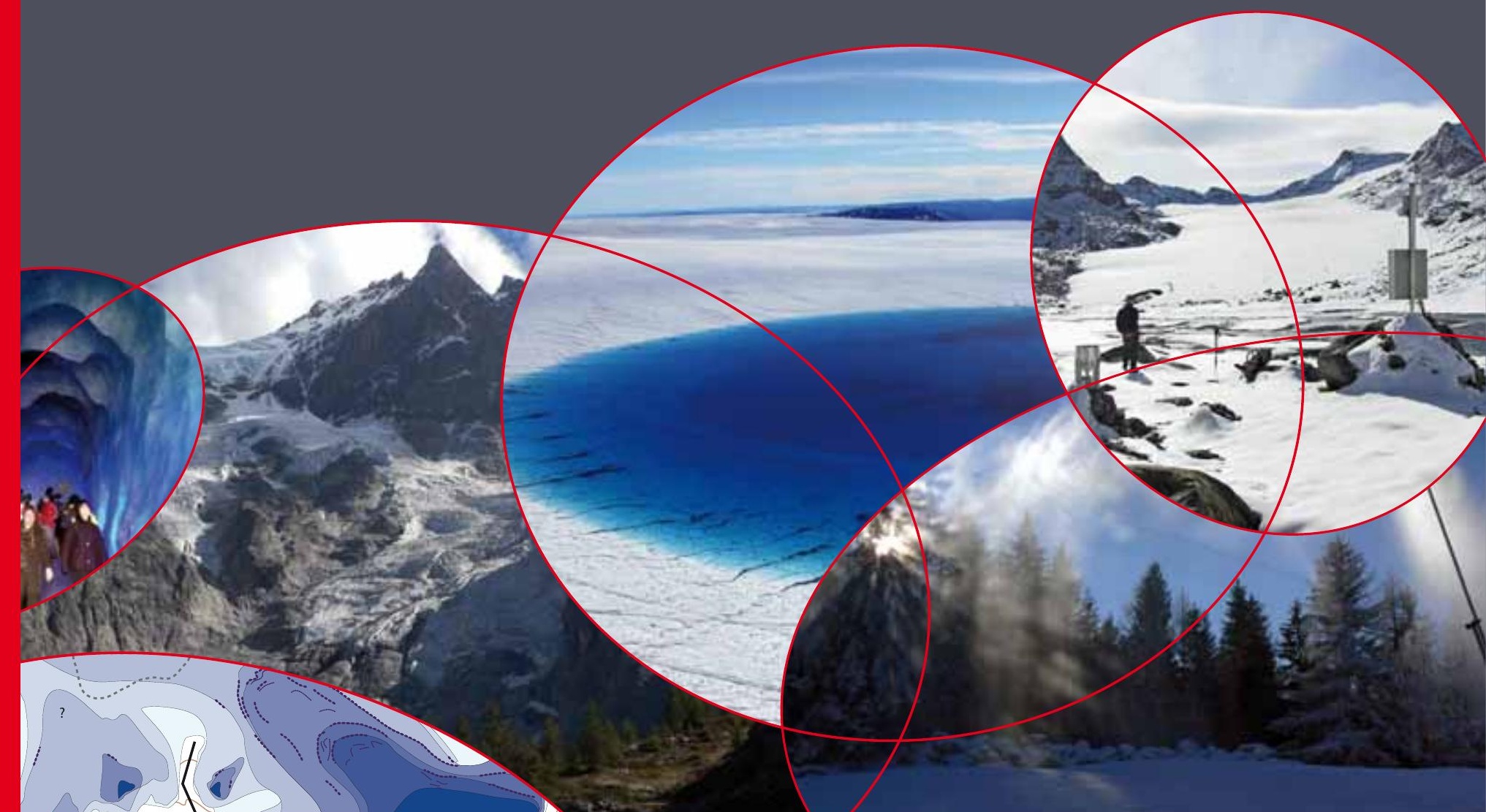





\title{
UN ÉLÉPHANT VOLANT EST-IL UN OISEAU?
}

\author{
PERSPECTIVES POUR L'OBSERVATION \\ HYDROMÉTÉOROLOGIQUE DES MILIEUX DE MONTAGNE
}

\author{
IS IT POSSIBLE TO TAKE A BIG-EARED FLYING ELEPHANT FOR A BIRD? \\ PERSPECTIVES FOR HYDRO-METEOROLOGICAL MONITORING OF MOUNTAINOUS CATCHMENTS.
}

Georges-Marie SAULNIER, ThIERry BARTH

Laboratoire EDYTEM, Université de Savoie/CNRS, Campus scientifique, F 73376 Le Bourget-du-Lac cedex.

Contact : georges-marie.saulnier@univ-savoie.fr

\begin{abstract}
RÉSUMÉ
L'hydrométéorologie dans les Alpes est largement sous-instrumentée. Environ $80 \%$ des pluviomètres installés dans les régions alpines $\left(40000 \mathrm{~km}^{2}\right)$ sont concentrés entre les altitudes 250 et 1250 mètres. Contrairement à ce que l'on pense couramment, les Alpes sont donc assez peu observées. Ceci est évidemment contradictoire avec la hausse des préoccupations sociales et environnementales qui se posent compte tenu des changements globaux très présents dans ces régions. Les raisons de ce sous-échantillonnage sont nombreuses. On peut citer par exemple la forte variabilité géographique et temporelle des variables météorologiques (difficulté à l'interpolation). Un autre frein est constitué par les coûts d'installation et de maintenance des appareils classiques qui doivent subir dans ces milieux de montagnes des contraintes pour lesquelles ils n'ont pas nécessairement été prévus (autonomie en énergie, rigueur des climats, phénomènes paroxysmiques : laves torrentielles, avalanches, etc.). Nous pensons qu'une nouvelle approche combinant l'instrumentation hydrométrique et la modélisation hydrologique physique peut être une des pistes permettant d'améliorer les bilans pluviométriques des milieux de montagne. Ce constat s'appuiera sur l'exposé d'une méthode originale d'analyse de la pertinence des modélisations hydrologiques ainsi que ses conséquences en termes de rétro-estimation des précipitations. Un réseau hydrométrique composé d'appareils novateurs, moins coûteux et adaptés aux conditions extrêmes (par exemple HyMAGE-TIP), couplé à des modélisations hydrologiques physiques doit permettre de soulager le cahier des charges d'un réseau de pluviomètres tout en obtenant un bilan hydrométéorologique correct et extensible en taille à des coûts moindres.
\end{abstract}

Mots-Clés : HYDROMÉTÉOROLOGIE, INSTRUMENTATION, MODÉLISATION HYDROLOGIQUE, ALPES.

\begin{abstract}
Hydrometeorology survey in the Alpes is clearly under-sampled. About $80 \%$ of rain-gauges installed in the Alpines regions $\left(40000 \mathrm{~km}^{2}\right)$ are concentrated within elevations ranging from $250 \mathrm{~m}$ to $1250 \mathrm{~m}$. Despite what is commonly thought, Alpes are actually little observed. This is obviously a contradiction with the increase of social and environmental questions raised by global changes, which are known to be very sensitive in these regions. Many reasons can explain such a under-sampling of the Alpine hydrometeorology. For example the high geographical and temporal variabilities of meteorological variables lead to uncertain interpolation. Another difficulty stands in the costly installations and maintenances of classical sensors that must face severe conditions for which they were not settled (energy autonomy, severe weather conditions, natural hazards such as debris flow, avalanche, etc.). It is suggested that a new approach combining hydrometry and physical hydrological modeling may be one of the answer leading to improve the water balance in mountainous regions. This will be illustrated by the explanation of a new method for hydrological models relevancy analysis that may have interesting ability of retro-identification of precipitations. A hydrometric network making intense use of new sensor, less costly and well suited for hazardous conditions (such as the HyMAGE-TIP sensor), coupled with physical hydrological models may give relief to the specifications of the rain-gauges network. Relevant hydro-meteorological balance should be then possible at "low" cost with possible geographical spread out.
\end{abstract}

KEYWORDS: HYDROMETEOROLOGY, SENSOR NETWORK, HYDROLOGICAL MODELING, ALPES.

Collection EDYTEM - n 8 - 2009 - Cahiers de Géographie 


\section{INTRODUCTION, PROBLÉMATIQUE}

L'observation de l'hydrologie et de la météorologie des milieux de montagnes est une tâche difficile. Il est possible d'évoquer un ensemble de raisons matérielles à ce constat : conditions d'accès physiques aux points de mesures, rigueurs du climat vis à vis des tolérances des matériels, autonomie en énergie souvent nécessaire de ceux-ci mais contraignantes à réaliser, peu d'accès aux télécommunications (hertziennes, IP, GSM, satellite, etc.) compte tenu de l'encaissement des vallées et talwegs, vulnérabilité des appareils (avalanches, crues/laves torrentielles, foudre, etc.). Mais on peut également évoquer un certain nombre de raisons liées à la physique de ces milieux. Par exemple, la forte variabilité spatiale des caractéristiques météorologiques (la pluie par exemple) rend hasardeuse l'interpolation de réseau de mesures discrètes et implique en conséquence la nécessité de réseaux de mesures denses et donc très onéreux. Il en va de même pour la couverture neigeuse qui, au moment de la fonte, montre de fortes hétérogénéités en fonction de son état (humide, froid, sec, etc.) l'exposition des versants, de leur disposition les uns par rapport aux autres (ombrages), de la couverture végétale, etc. Egalement, la géologie superficielle et la géomorphologie ne sont pas souvent aisées à cartographier sur des surfaces de quelques milliers de $\mathrm{km}^{2}$ et ajoutent encore à l'incertitude de la représentativité des réseaux de mesures actuels. Pour toutes ces raisons, force est de constater que notre observation hydrométéorologique des milieux de montagnes est encore largement insuffisante.

Pour le cas des Alpes françaises par exemple (superficie supérieure à $40000 \mathrm{~km}^{2}$ ), seulement une soixantaine de stations pluviométriques de durée significative sont disponibles (Kieffer et Bois, 1997). A titre de comparaison (Le Lay et Saulnier, 2007), on dénombre environ 400 pluviomètres journaliers et 180 pluviomètres horaires sur la région Cévennes-Vivarais (Cèze, Ardèche, Gardons : $4500 \mathrm{~km}^{2}$ ). De plus, ce réseau de pluviomètres alpins est biaisé puisqu'il se répartit entre 75 et 1780 mètres d'altitudes et qu'environ $80 \%$ de ces pluviomètres sont concentré entre les altitudes 250 et 1250 mètres (Kieffer et Bois, 1997).

Concernant l'hydrologie des cours d'eau alpins, le constat est proche. Pour exemple on peut consulter la carte de vigilances crues (http://www.vigicrues.ecologie.fr) : quatre stations sur les Alpes du Nord font l'objet d'une prévision des débits en temps réel contre plus d'une vingtaine sur une surface bien moindre en région Cévennes-Vivarais. Bien entendu, cette comparaison est très critiquable puisqu'elle n'évoque pas l'énorme différence entre les risques d'inondations des territoires alpins et cévenols, ni l'influence des ouvrages hydroélectriques sur la régulation de certains cours d'eau alpins.

Ces critiques ne sauraient toutefois remettre en cause le constat d'une sous-instrumentation hydrométéorologique des milieux de montagnes alpins. Ce constat est d'autant plus évident quand on tente de collecter des informations quantitatives pour entreprendre des recherches hydrométéorologiques dans ces régions. A ce titre d'ailleurs, on peut souligner l'absence de bases de données hydrométéorologiques disponibles décrivant dans la durée un territoire de montagne de taille significative. Les données sont souvent la propriété d'organismes dont on peut très bien imaginer, sans nécessairement les partager toutes, les raisons pour lesquelles ils ont des difficultés à les diffuser facilement.

Dès lors, comment envisager d'améliorer la densité (que l'on confondra ici avec la représentativité) de l'observation hydrométéorologique dans le milieu alpin ?

Une réponse simple mais coûteuse comme celle proposant de multiplier le nombre de pluviomètres dans les Alpes ne peut être la seule réponse envisageable à l'heure actuelle. L'installation de réseaux de pluviomètres est une nécessité mais ils ne seront malheureusement probablement jamais installés en nombre suffisant pour échantillonner correctement la variabilité spatio-temporelle des précipitations (sous formes liquide ou solide). L'utilisation des réseaux radars, qu'ils soient de longue portée (radars bande $\mathrm{C}$ du réseau de Météo-France par exemple) ou à courte portée comme les radars à bande $\mathrm{X}$, n'est pas non plus une réponse évidente compte tenu des coûts et des perturbations qu'induit le fort relief de ces régions sur le signal radar (effets de masques, bandes brillantes, etc.).

De plus les moyens humains et financiers ne sont pas illimités et des questions comme celle de l'élaboration de descriptions pertinentes du cycle de l'eau en milieux de montagnes et de son interaction avec les changements globaux et les sollicitations socio-économiques constituent des urgences sociétales. Il est donc nécessaire d'imaginer maintenant des approches nouvelles et complémentaires pour améliorer le suivi hydrométéorologique en milieux de montagne. Cet article se propose d'en explorer une.

En effet, dans la suite de cette communication, nous présentons une méthode permettant de réduire les effets d'un constat d'échec des modélisations hydrologiques que nous expliquons également. Nous dessinerons ensuite un certain nombre de perspectives faisant usage de cette méthode et qui, selon nous, pourraient contribuer à envisager une nouvelle façon de construire le suivi hydrométéorologique d'une vallée étudiée. Cette approche couple véritablement l'élaboration de la donnée et la modélisation de la variable mesurée et peut contribuer, nous l'espérons, à permettre un suivi robuste et rapide de l'hydrométéorologie en montagne à des coûts non prohibitifs. 


\section{I - L'IMPASSE ACTUELLE DANS L'USAGE DE NOS MODÉLISATIONS HYDROLOGIQUES}

\section{1 - Problème posé par la calibration des modèles numériques}

Les modélisations hydrologiques sont un outil d'aide à la compréhension, à la description et la représentation de nos connaissances sur le fonctionnement des bassins versants. Quel que soit le type de ces modèles, empiriques ou physiques, des calibrations sont nécessaires pour spécifier les valeurs de leurs paramètres non mesurables sur le terrain. Les raisons en sont multiples. Par exemple, elles peuvent être d'origines conceptuelles : certains paramètres ne sont pas directement liés à des caractéristiques physiographiques du bassin comme par exemple un coefficient de vidange d'un réservoir d'un modèle conceptuel. Quelques fois, les échelles de conceptualisation et d'instrumentation ne sont pas compatibles : comment mesurer l'épaisseur de sol " équivalente » d'un pixel de $250 \mathrm{~m}^{2}$ d'un Modèle Numérique de Terrain (MNT) ? Souvent la taille des bassins étudiés rend impossible la mesure exhaustive de certains paramètres : comment cartographier, à la résolution de sa variabilité spatiale, la conductivité hydraulique des sols d'un bassin de taille supérieure à $100 \mathrm{~km}^{2}$ ?

La calibration consiste alors à tenter d'estimer la valeur de ces paramètres non mesurables par tâtonnement, en cherchant à rapprocher le plus possible la simulation faite par le modèle de certaines variables pronostiques (par exemple le débit) en fonction des variables de forçages (par exemple la pluie). Ces «tâtonnements » sont bien entendu effectués la plupart du temps par des méthodes numériques complexes, systématiques et qui constituent un corpus de recherches méthodologiques autonomes.

Une conséquence bien connue de cette étape de calibration est que les valeurs des paramètres estimées de cette façon peuvent prendre des valeurs très différentes de leurs « vraies » valeurs (celles que l'on pourrait mesurer sur le terrain si cela était possible). L'étape de calibration cherche en effet à maximiser la ressemblance entre la simulation du modèle et les observations choisies pour l'optimisation, quitte à tordre la physique du modèle. Ainsi, rien n'empêche la calibration de forcer le modèle à adopter un comportement extrême ou irréaliste, par comparaison à ce que l'on observe sur le terrain ou par rapport aux hypothèses mêmes du modèle, si cela permet d'obtenir les calculs les plus précis possibles des quelques variables pronostiques optimisées par la calibration (d'ailleurs souvent réduites à une seule : le débit à l'exutoire du bassin). Ceci a des conséquences dommageables dans la validation des modélisations hydrologiques (même si le terme validation est encore le plus courant, on préférera dans la suite celui de corroboration).

En effet, quand on cherche à corroborer un modèle pluie-débit par exemple, c'est-à-dire à estimer sa pertinence pour un bassin versant, on compare en général son aptitude à correctement calculer les débits à certains points jaugés du réseau hydrographique, en fonction des pluies observées par rapport aux débits observés. On souhaiterait donc qu'un modèle à la physique inappropriée pour le bassin versant étudié fournisse de « mauvais » débits calculés et qu'un modèle à la physique cohérente avec la physiographie du bassin fournisse de « bons » débits calculés. C'est l'attente la plus légitime et qui devrait permettre de faire progresser nos modélisations en rejetant les mauvaises et en jugeant les progrès réalisés par nos recherches. Mais la calibration rend totalement incertaine cette attente.

\section{2 - Un éléphant bien entrainé a les mêmes aptitudes au vol qu'un oiseau}

Pour illustrer ce propos nous présentons l'utilisation de deux modèles : le modèle Dumbo et le modèle Oiseau. Ces deux modèles, dont nous ne donnerons volontairement les détails que plus tardivement dans cet article, ont des hypothèses de fonctionnement hydrologique opposées.

L'un fonde son hypothèse de génération du ruissellement sur un concept plutôt Hortonien (Horton, 1933). Ce type de ruissellement apparait lorsque les intensités de pluie dépassent la capacité d'infiltration des sols : «le sol ne boit pas assez vite l'eau des pluies». Dans ce cas, l'excédent stagne à la surface du sol et peut s'écouler sous l'effet de la pente. C'est le type de ruissellement le plus naturellement compris (et donc le plus étudié), observé (car il est visible à l'échelle de l'homme : quelques $\mathrm{m}^{2}$ ) et intuitif (ce qui achève d'en faire la base des cours d'hydrologie). Ce type de ruissellement est par exemple prépondérant dans les bassins aux sols compacts, peu pentus, faiblement végétalisés, aux climats plutôt arides (figure 1). Mais d'une façon générale ce type de ruissellement apparaît quand la texture du sol est la variable principale contrôlant le taux d'infiltration des précipitations.

L'autre modèle fonde son hypothèse de génération du ruissellement sur le concept Hewlettien (Hewlett et Hibbert, 1967 ; Cappus, 1960). Dans ce cas, les sols sont au contraire généralement capables d'infiltrer de grands volumes de précipitations et de grandes intensités de pluie. La capacité d'infiltration des sols n'est donc pas ici le facteur limitant. En effet, ce type de ruissellement est plutôt prépondérant dans les bassins aux sols végétalisés, faiblement ou fortement pentus, aux climats tempérés (figure 2). D'une façon générale, ce type de ruissellement apparaît quand la structure du sol permet de fait de grands taux d'infiltration. Dans ces cas, les pluies s'infiltrent en quasi-totalité et s'écoulent dans les premiers mètres du sol sous l'effet des pentes. Le long des versants, localement, le profil de sol peut par contre se gorger d'eau (rupture de pente, surplus d'eau drainée de la partie amont du versant). La précipitation tombée 

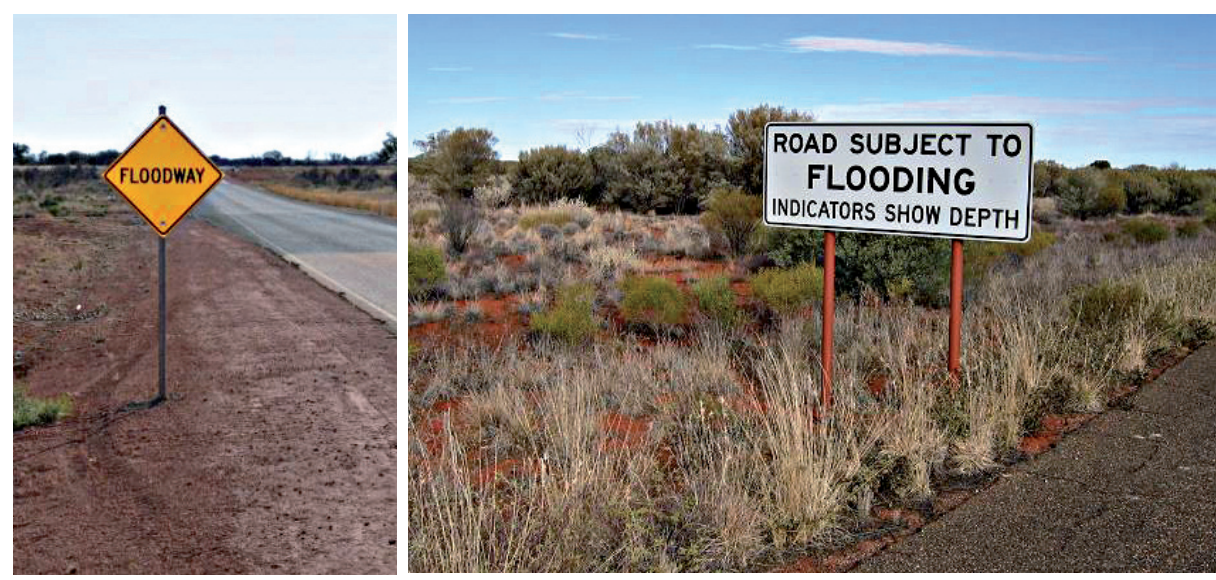

Figure 1 - Exemples de sols générant principalement $d u$ ruissellement Hortonien (Australie; photos G.M.Saulnier).

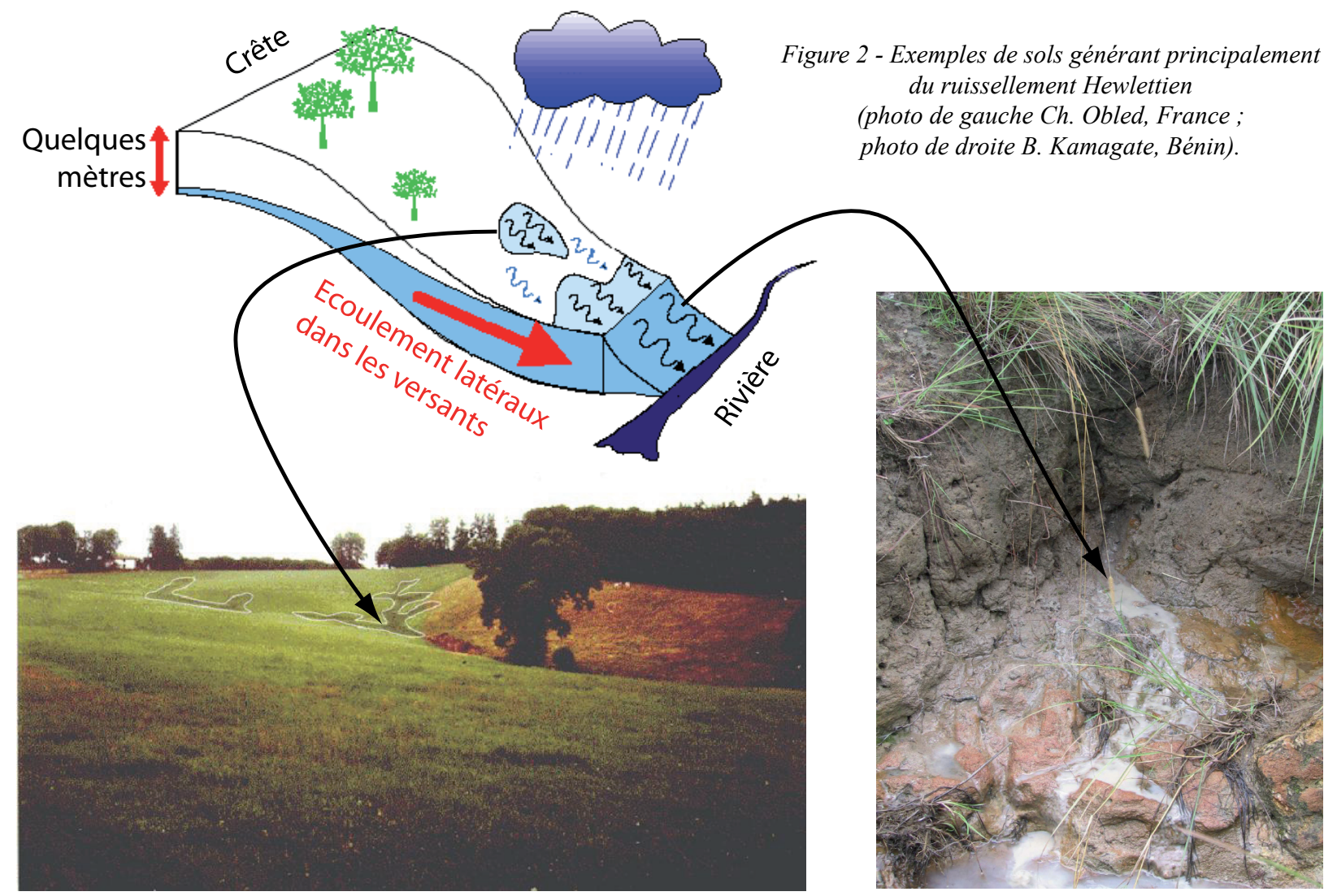

en ces endroits saturés ne peut alors plus s'infiltrer et s'écoule alors sous forme de ruissellement rapide jusqu'à la rivière.

Dans le fonctionnement Hewlettien, ce n'est plus « une fraction de la pluie qui ruisselle sur une très grande partie du bassin » (cas Hortonien) mais « une grande partie de la pluie qui ruisselle sur une fraction du bassin ». L'état d'humidité du bassin est le facteur prépondérant dans la genèse du ruissellement Hewlettien alors que dans le cas Hortonien ce sont les valeurs fortes des intensités des pluies. Si un bassin est Hewlettien, la structuration de ses sols suppose une forte capacité d'infiltration et donc la presque absence de ruissellement Hortonien (au-delà de quelques $\mathrm{m}^{2}$ ). À l'inverse, dans un bassin Hortonien, les sols sont compacts et présentent de faibles structurations qui rendent négligeable la formation de ruissellement sur surfaces saturées. C'est à ce titre que l'on peut qualifier les hypothèses de ruissellement Hortonien et Hewlettien de contradictoires.

Le modèle Oiseau et le modèle Dumbo, représentant chacun un de ces deux processus de génération du ruissellement, ont exactement le même nombre de paramètres à caler (deux). Nous omettons volontairement leurs descriptions pour le besoin pédagogique de la démonstration. Le transfert du ruissellement jusqu'à la rivière ainsi que la propagation en rivière sont calculés grâce à une fonction de transfert de type Hydrogramme Unitaire estimée préalablement par la méthode Différence Première de la Fonction de Transfert (D.P.F.T. : Duband et al., 1993). Le but n'est pas ici de justifier les choix 
algorithmiques de cet exercice mais de s'assurer que :

- la complexité des deux modèles est identique. Cette complexité est ici exprimée par le nombre de paramètres à caler, c'est-à-dire par leur nombre de degrés de liberté ;

- les processus de transfert sont résolus de façon identique pour les deux modèles et par une méthode qui ne nécessite pas d'hypothèse préalable sur le processus de ruissellement. Le transfert est donc indépendant des hypothèses de génération du ruissellement des deux modèles et leur est commun.

Ainsi, les différences constatées par la suite ne sont imputables qu'aux hypothèses différentes de fonction-

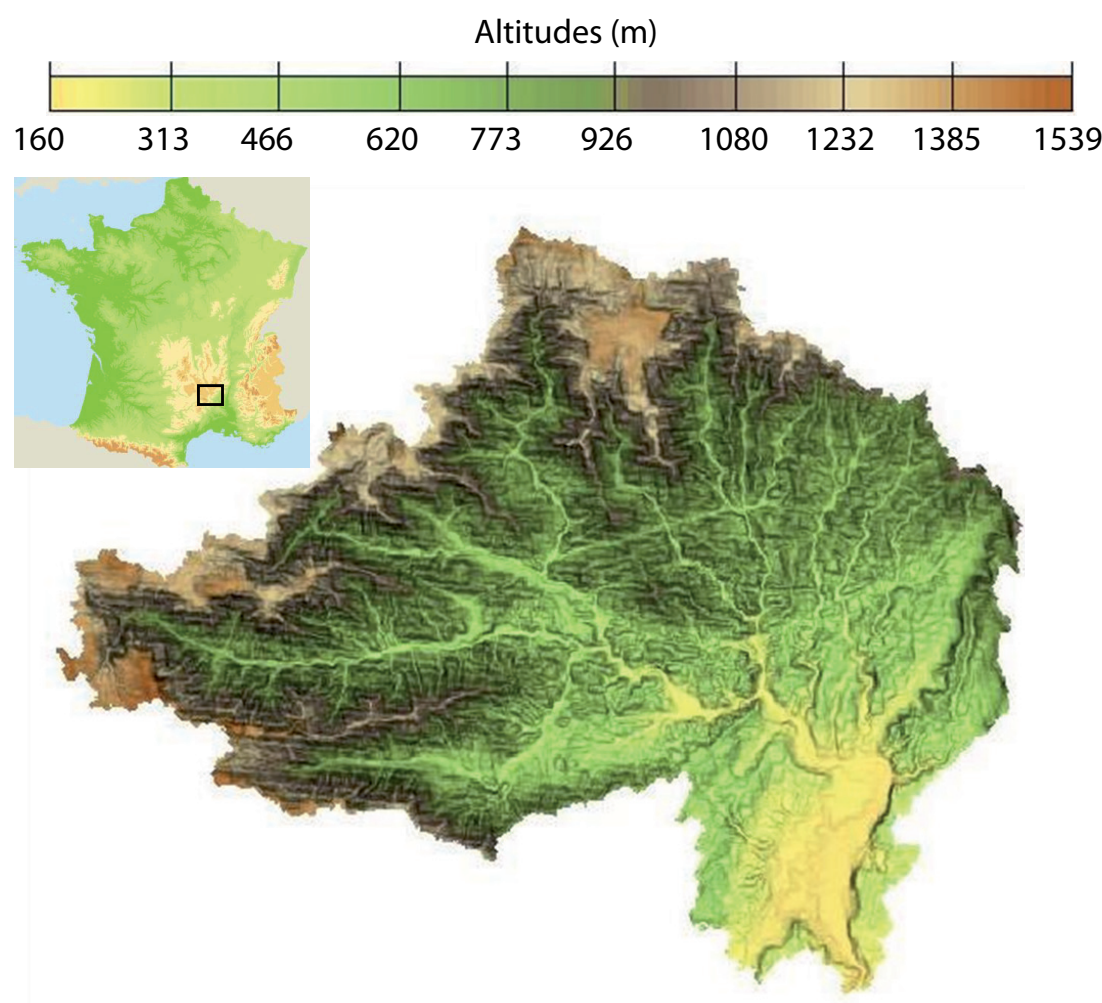

Figure 3 - Le bassin de l'Ardèche à Vogüe $\left(640 \mathrm{~km}^{2}\right.$, Ardèche, France).

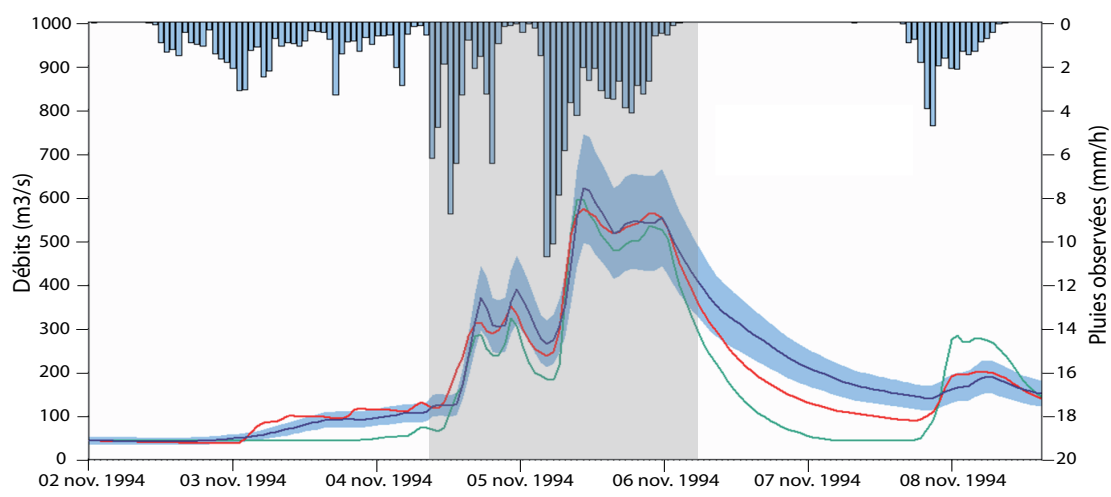

Figure 4 - Simulations par les modèles Dumbo (en vert) et Oiseau (en rouge) de l'épisode du 2 novembre 1994 à Vogüe. Comparaisons aux débits observés (en bleu). nement des bassins versants proposées par les deux modèles.

Les deux modélisations ont donc été calées sur un bassin versant ardéchois : le bassin de l'Ardèche à Vogüe $\left(640 \mathrm{~km}^{2}\right.$, figure 3$)$. La figure 4 superpose les débits calculés par le modèle Oiseau et par le modèle Dumbo ainsi que les débits mesurés à la station de jaugeage de Vogüe. L'évènement considéré est celui du 2 novembre 1994. Il s'agit d'un évènement intense mais non extrême : les processus de genèse du ruissellement ont donc ici une importance grande au contraire d'un évènement paroxysmique où les valeurs des pluies contrôlent l'essentiel des valeurs de débits. Ces derniers sont encadrés à titre indicatif par une bande d'incertitudes de $20 \%$ qui représente le minimum d'incertitude que l'on peut attendre sur ces bassins cévenols très difficiles à jauger.

On peut exprimer un certain nombre de remarques à la lecture de ce graphe :

- les deux modèles sont de façon quasi synchrone à l'intérieur ou à l'extérieur de la bande d'incertitude (à part pour les faibles débits où le ruissellement est négligeable dans la valeur du débit). Vis-à-vis de la génération du ruissellement ces deux modèles devraient donc être « acceptés » pendant la phase de crue autant l'un que l'autre ;

- les alternances montée de crue et décrue sont similaires pour les deux modèles. La montée au pic de crue est très bonne pour les deux modèles ;

- le pic de crue est légèrement mieux simulé par le modèle Dumbo ;

- le modèle Dumbo est désavantagé sur cet épisode pour les faibles débits et lors de la seconde remontée des débits en fin d'épisode. Il est juste toutefois de préciser que sur d'autres évènements de la base de données dont nous disposons le cas inverse est quelque fois observé ;

- globalement, les deux modèles se comportent de façon similaire compte tenu des incertitudes de mesures des débits et présentent le même 
intérêt pour la prévision des crues en temps réel. Notons d'ailleurs que le modèle Dumbo est de la même famille que le modèle actuellement utilisé par le «Service de Prévision des Crues du GrandDelta» (organisme en charge de la prévision des inondations en temps réel : http://www.gard.equipement.gouv.fr).

Si les deux modèles sont aussi performants l'un que l'autre dans la phase de ruissellement, cela pose le problème de la corroboration des modélisations puisqu'ils ont des hypothèses de genèse du ruissellement contradictoires. Comment dire que l'un des modèles a une physique plus cohérente avec la réalité physiographique de ce bassin alors que leurs débits calculés ne diffèrent pas significativement dans la phase de crue (i.e. là où le ruissellement est prépondérant) ? On constate ici à quel point la calibration des paramètres permet de rapprocher les débits simulés par un modèle des débits observés, mais au prix de la perte de la possibilité de critiquer objectivement les hypothèses du modèle.

\section{II - DEVINER LES PLUIES A PARTIR DES SEULS DEBITS}

\section{1 - La proposition}

La méthode originale que nous proposons pour solutionner cette difficulté repose sur une idée simple : les débits d'un bassin sont la combinaison (complexe) entre la physique de ce bassin et la chronologie des précipitations.

Cette physique de bassin, les deux modèles pensent en avoir une idée puisqu'elle fonde leurs hypothèses. Si nous « cachons » les pluies à un modèle est-il capable d'utiliser ses propres hypothèses sur le fonctionnement du bassin afin de « deviner » la chronologie des précipitations?

En ne fournissant ainsi comme seule information au modèle la chronologie des débits, celui-ci devra compter sur sa seule physique pour reconstituer la chronologie des précipitations qu'il juge nécessaire pour reproduire les débits observés. Si nous parvenons à lui faire faire ce travail, nous pourrons comparer les pluies reconstituées par le modèle et les comparer aux pluies réelles. Puisque les modèles Dumbo et Oiseau ont des hypothèses différentes, nous pouvons alors nous poser deux questions :

- vont-ils reconstituer des chronologies de pluies suffisamment différentes et sans équivoque cette fois pour que l'on puisse distinguer l'effet de leurs hypothèses contradictoires?

- un des deux modèles réussit-il à correctement reconstituer les précipitations, ce qui validerait a posteriori son hypothèse hydrologique?

Dans cette démarche, seuls les débits sont fournis aux modèles. Ceux-ci n'auront donc pas la possibilité de faire prendre des valeurs irréalistes à leurs paramètres pour compenser leurs erreurs de raisonnement puisqu'ils n'auront pas accès à la connaissance sur les pluies. Cette démarche aurait ainsi l'avantage d'éviter le biais de la calibration illustré précédemment.

\section{2 - La méthode}

La méthode que nous proposons pour faire « inverser » la chronologie des pluies par un modèle quelconque à partir des seules valeurs de débits est un processus itératif. L'idée de base consiste à considérer au départ les pluies toutes nulles puis à les incrémenter petit à petit. Au départ, les débits que peut calculer le modèle à partir de ces pluies nulles est donc la version la plus sous-estimée possible du débit : celle où rien ne se passe à part une récession continue des valeurs de débits calculés (figure 5, étape A). Les débits calculés doivent donc être tous inférieurs ou égaux aux débits observés. En augmentant progressivement les valeurs de pluies, on doit toujours respecter cette condition puisque l'on choisit délibérément de partir de pluies sous-estimées.
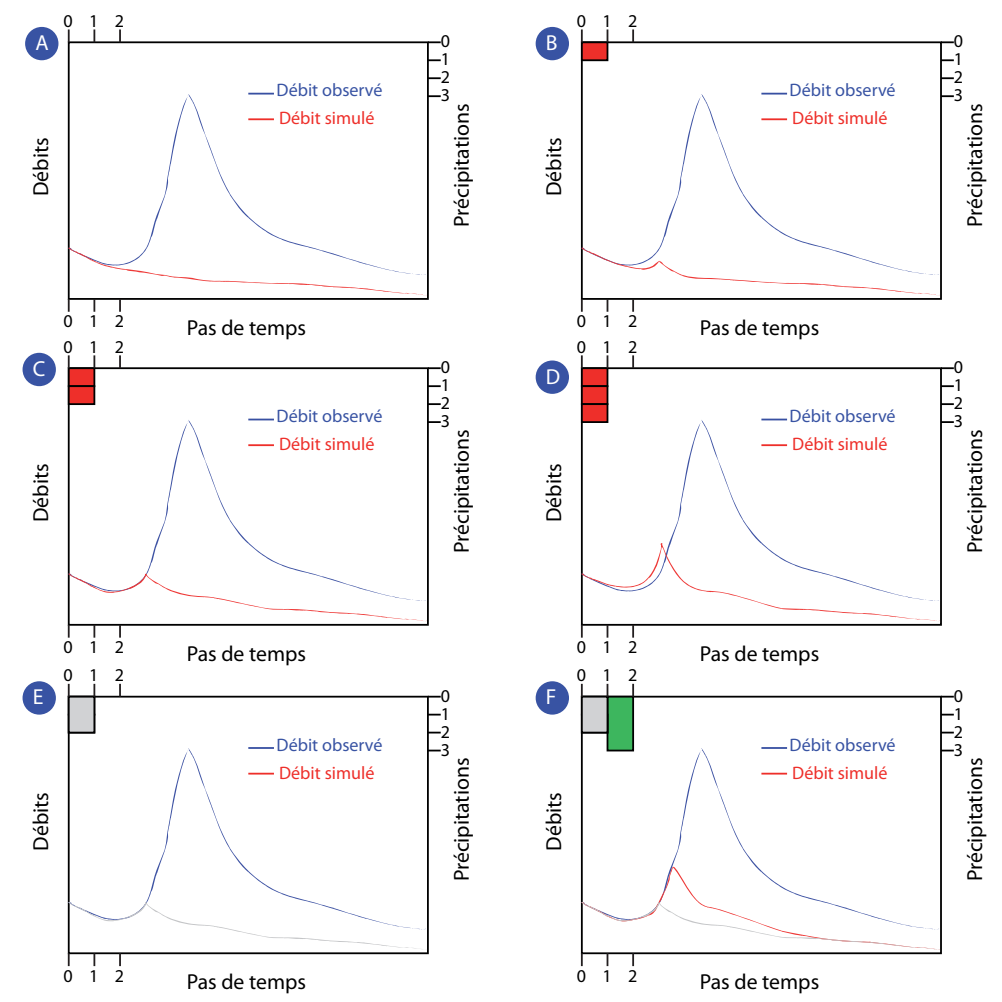

Figure 5 - Etapes de la méthode d'inversion de la chronique des pluies moyennes pour l'évènement du 2 novembre 1994 à Vogüe. 
On cherche donc, en augmentant progressivement les valeurs des précipitations, à « remonter » les débits calculés jusqu'à coller le plus possible les débits observés. Le postulat « les débits calculés sont tous inférieurs ou égaux aux débits observés » est la condition logique centrale de l'algorithme.

Après avoir ainsi initialisé les pluies à des valeurs nulles, on s'intéresse aux valeurs de pluies pas de temps par pas de temps, du premier jusqu'au dernier. Pour le premier pas de temps de la série de pluie à deviner, on peut maintenant se poser la question de savoir si la pluie est effectivement «probablement nulle» ou «probablement non nulle ». Pour ce faire, on incrémente la valeur de pluie du premier pas de temps d'une valeur fixée, par exemple $1 \mathrm{~mm} / \mathrm{h}$. En refaisant une simulation à cette étape, les pluies devinées sont constituées d'un seul créneau de $1 \mathrm{~mm} / \mathrm{h}$ au premier pas de temps et de pluies toutes nulles le reste du temps (figure 5, étape B). Ce scénario est donc encore un scénario sousestimé puisque l'on considère l'arrêt des pluies après le premier pas de temps. Les débits calculés doivent donc être encore une fois tous inférieurs ou égaux aux débits observés. Si tel est le cas, on peut supposer que l'on a sous-estimé la pluie du premier pas de temps. Incrémentons donc encore une fois cette valeur qui vaut maintenant $2 \mathrm{~mm} / \mathrm{h}$ (figure 5 , étape C).

On itère ainsi jusqu'à ce que les débits calculés ne respectent plus la condition nécessaire d'être tous inférieurs ou égaux aux débits observés. Quand cette condition n'est plus remplie c'est que l'on vient de dépasser la valeur probable de pluie au premier pas de temps (figure 5, étape $\mathrm{D}$ ). On revient donc à la dernière valeur testée pour le premier pas de temps que l'on fixe cette fois « dans le marbre » pour le reste de l'exécution de la méthode (figure 5, étape E).

On peut s'intéresser maintenant au deuxième pas de temps et refaire pour lui ce qui a été réalisé pour le premier pas de temps (qui a gardé sa valeur devinée) (figure 5, étape F). On itère ainsi pour tous les pas de temps de la série de pluie à deviner.

Pour calculer les débits à partir de cette reconstitution itérative des précipitations, le modèle a bien entendu besoin de connaître les valeurs des paramètres présents dans ses équations. Mais dans notre méthode, il ne les connaît pas et n'a pas de moyens de les fixer puisque nous l'empêchons d'user de la calibration. La méthode proposée va donc imposer au modèle de reconstituer les précipitations pour l'ensemble des combinaisons possibles de valeurs des paramètres du modèle. Dans les faits, seule une partie de ces combinaisons est testée : celle permettant d'échantillonner correctement (d'un point de vue statistique) l'espace possible de ces combinaisons de paramètres. Comme on le devine, cette méthode est donc très intensive d'un point de vue des calculs et ne fonctionnera que pour des modèles hydrologiques ayant des performances numériques suffisamment bonnes, ce qui de toute façon est une condition nécessaire de nos jours pour un modèle digne de ce nom (i.e. capable de simuler ses incertitudes, de recevoir en entrée des pluies bruitées, de réaliser de l'assimilation de données, etc.).

Dans l'exemple qui suit, par souci de clarté, nous ne présenterons pas l'ensemble des résultats de cette méthode appliquée aux deux modèles présentés plus haut. Nous présentons uniquement la reconstitution des précipitations donnant la meilleure proximité entre les débits calculés à partir de ces pluies inversées et les débits observés.

\section{3 - Voler n'est pas un critère suffisant pour qu'un éléphant puisse être considéré comme un oiseau}

Appliquée aux modèles Dumbo et Oiseau cette méthode fournit les résultats illustrés sur la figure 6 . Sur cette figure sont comparées pour le modèle Dumbo et le modèle Oiseau les pluies réellement observées (vert) et les pluies devinées (bleu) par les modèles pour l'événement de crue du 2 novembre 1994 déjà présenté.

Il est intéressant de remarquer les différences bien davantage marquées cette fois entre les deux réalisations des modèles. Les allures des pluies reconstituées par les deux modèles amènent quelques remarques :

- le modèle Oiseau détecte très correctement les débuts et fin d'épisodes pluvieux et ce même pour l'averse en fin d'événement, malgré une interruption significative des pluies les 6 et 7 novembre 1994 ;

- le modèle Oiseau reconstitue des valeurs d'intensités maximales très proches de celles observées : $8,5 \mathrm{~mm} / \mathrm{h}$ contre $8,75 \mathrm{~mm} / \mathrm{h}$ en début d'après-midi du 4 novembre $1994,10 \mathrm{~mm} / \mathrm{h}$ contre $10,7 \mathrm{~mm} / \mathrm{h}$ en fin de matinée du 5 novembre 1994 ;

- le modèle Oiseau représente relativement correctement l'amplitude de variation des précipitations entre l'accalmie des pluies à la fin du 4 novembre 1994 et leur reprise en début de journée du 5 novembre 1994 ;

- le modèle Dumbo présente des pluies respectant les alternances générales de hausses et de décroissances des intensités des pluies ;

- par contre l'allure des pluies reconstituées est bien plus mauvaise que celles du modèle Oiseau avec des intensités très sous-estimées et très autocorrélées ;

- l'interruption des précipitations des 6 et 7 novembre 1994 est complètement ratée.

Les deux modèles surestiment légèrement les précipitations réellement observées. Le modèle Oiseau reconstitue un cumul de $230 \mathrm{~mm}$ contre $212 \mathrm{~mm}$ observés (soit une surestimation de $8,5 \%$ ) et le modèle Dumbo reconstitue un cumul de $247 \mathrm{~mm}$ (soit une surestimation de $16,3 \%$ ).

L'exercice proposé est encourageant. En effet, au contraire de la comparaison floue des modèles après calibrations, les résultats de leurs reconstitutions des 

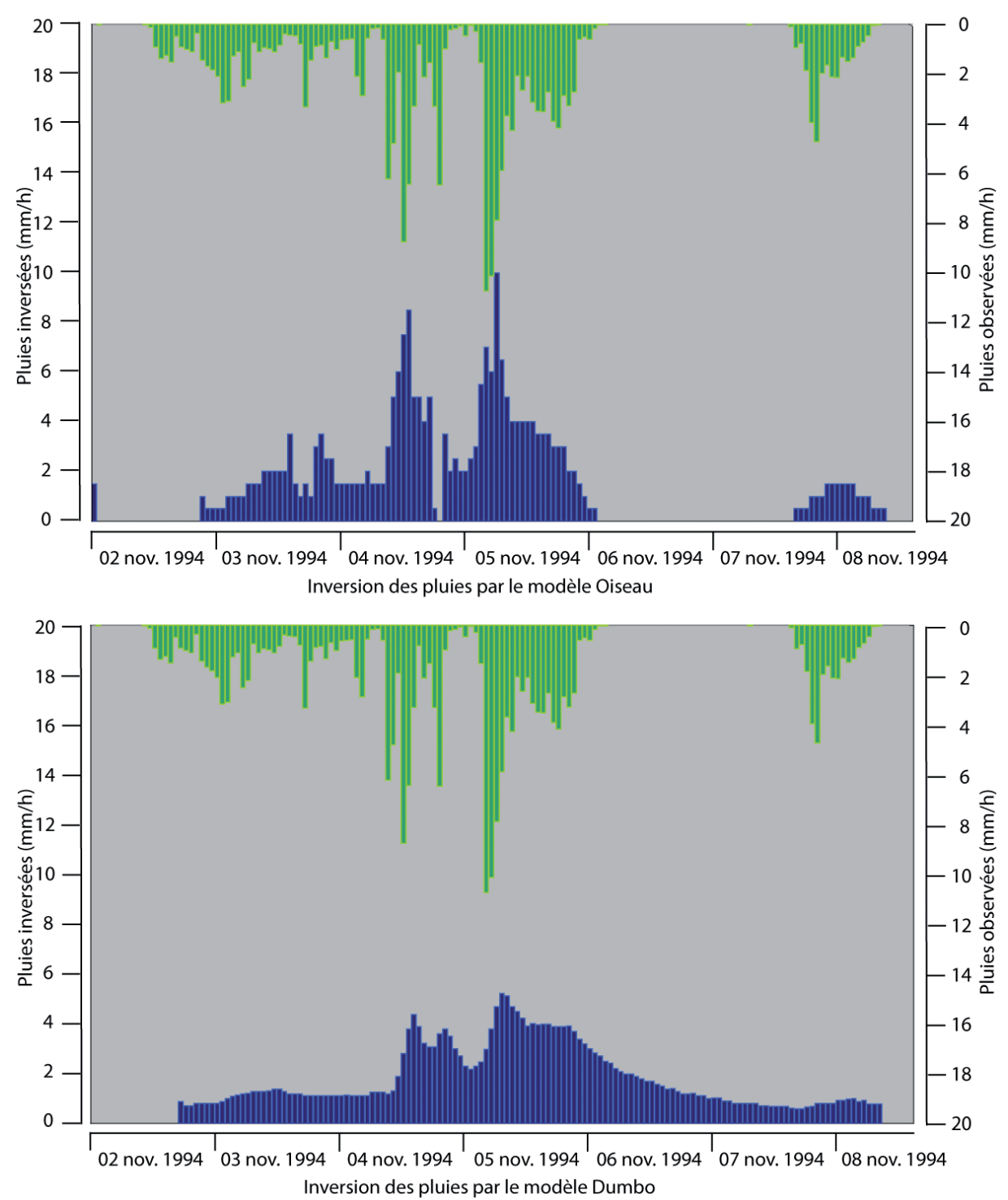

Figure 6 - Résultats de la méthode d'inversion des pluies appliquée aux modèles Dumbo et Oiseau pour l'évènement du 2 novembre 1994 à Vogüe.

sible de juger de la qualité de ces hypothèses en comparant les résultats avec des mesures de terrain. Ainsi, le modèle Oiseau peut être considéré comme pertinent pour la modélisation de ce bassin cévenol au contraire du modèle Dumbo alors que, rappelons-le, la classique comparaison de leurs performances en termes de reconstitution des débits après calibration ne le permettait pas rigoureusement. A ce titre, nous espérons que la méthodologie proposée constitue une piste sérieuse pour résoudre une partie de l'impasse actuelle de nos modélisations énoncée plus haut.

Pour informations, le modèle Oiseau est une version du modèle hydrologique TOPMODEL (TOPography-based MODEL, Beven et Kirkby, 1979 ; Beven et al., 1995) déclinée dans une version simplifiée (Saulnier et al., 1998 ; Saulnier et Datin, 2004). Le modèle Dumbo est le classique modèle SCS-CN (Soil Conservation Service, 2003). Les TOPMODELs sont très bien adaptés pour

pluies à partir des seuls débits conduit à pouvoir les discriminer sans ambigüité aux regards des allures de leurs pluies reconstituées. Deux modèles aux hypothèses physiques différentes fournissent donc des simulations différentes sans que ce résultat soit biaisé par les travers de la calibration. Qui plus est, il est pos- la représentation des écoulements de subsurfaces dans les versants et la genèse du ruissellement sur surfaces contributives (processus Hewlettien) alors que le SCS, qui calcule principalement le ruissellement en fonction des précipitations reçues, s'apparente à une représentation du ruissellement par le processus Hortonien.

\section{III - QUELLES PERSPECTIVES POUR L'INSTRUMENTATION HYDRO-METEOROLOGIQUE DES MILIEUX DE MONTAGNES?}

La transition première est la suivante : on constate que la reconstitution des pluies est capable de donner des résultats corrects et ce d'autant plus que le modèle est proche de la physique du bassin puisqu'à l'inverse un modèle inadapté au bassin fournira des reconstitutions de pluies fausses. C'est donc un encouragement et une approche plus saine de la recherche en modélisation hydrologique qui ne calerait plus les modèles pour les rendre précis mais les améliorerait pour les rendre justes.

S'il devient enfin possible de critiquer les hypothèses physiques des modèles sans biais et d'améliorer 
ainsi leurs pertinences on peut imaginer disposer de modélisations hydrologiques des milieux de montagnes de plus en plus physiques. Dans ce cas, pourquoi ne pas faire usage de ces modélisations physiques et justes pour accompagner l'instrumentation si difficile en milieux de montagnes?

Comme on l'a expliqué, augmenter le nombre de capteurs du réseau de mesure de façon considérable (par rapport à l'existant) et suffisamment dense (par rapport à la forte variabilité spatiale de l'hydrométéorologie de montagne) est un but louable et de long terme mais inaccessible pour encore de nombreuses années compte tenu des moyens que cela engagerait. Parmi toutes les difficultés, la plus incontournable est probablement celle de la mesure dense et précise des précipitations en montagne. Pour répondre à cet écueil, la démarche " fusionnelle » entre instrumentation et modélisation que nous proposons pourrait s'articuler en trois points :

\section{1 - L’hydrométrie}

Un certain nombre de capteurs hydrométriques robustes, peu onéreux et adaptés aux milieux/conditions extrêmes voit le jour. Par exemple, EDYTEM, en collaboration avec un certain nombre de partenaires opérationnels (SCHAPI, Service de Prévision des Crues du Grand-Delta), développe un capteur hydrométrique basé sur l'utilisation de caméras vidéos (visible et infrarouge) nommé HyMAGE-TIP (http://www.hydro-meteo.fr). Ce capteur a l'avantage de ne pas être immergé dans le lit des torrents de montagne et de pouvoir être placé à bonne distance de ceux-ci. Il est donc beaucoup moins vulnérable que les capteurs hydrométriques classiques. Il a donc des conditions de fonctionnement indépendantes de l'état du torrent (crues/laves torrentielles). De plus, il présente des précisions de mesures fonction des propriétés géométriques de la scène filmée (contrôlables) et non des conditions hydrauliques des torrents (incontrôlables).

S'il est ainsi possible d'instrumenter dorénavant l'hydrométrie des cours d'eau de montagnes de façon relativement économe et robuste, il est aussi possible d'imaginer fonder un réseau hydrométéorologique de montagnes équipé par ces capteurs hydrométriques originaux de façon dense. Comme ces capteurs mesurent les débits, ils sont généralement installés plus en aval des torrents de montagne, ce qui correspond souvent à la présence proche de collectivités (i.e. infrastructures, énergies proches, relais de télécommunications proches) et des conditions d'accessibilité plus aisées que les versants sommitaux des montagnes. De plus, leurs mesures représentent un bilan hydrologique des bassins amonts qu'il est plus aisé de contraindre que celui de l'entrée des précipitations liquides ou neigeuses très variables dans l'espace. En effet, celles-ci sont dispersées sur un espace bien plus grand (la surface du bassin versant) que celui de la mesure de débit (la section transversale du lit du torrent à son exutoire mesuré).

\section{2 - La modélisation}

L'utilisation de modélisations physiques que l'on aura pris soin de rendre cohérentes avec la physiographie des régions étudiées (géomorphologie, géologie, hydrologie de surface, couvert végétal) peut permettre grâce à la méthode que l'on a présentée plus haut de reconstituer des chroniques de précipitations à partir des mesures de débits que l'on aurait réalisées. Toutefois, comme nous l'avons précisé plus haut, plusieurs reconstitutions de précipitations peuvent être produites par la méthode pour un modèle donné puisque les valeurs des paramètres de celui-ci ne sont pas connues. Dans l'exercice précédent, nous n'avons présenté que la meilleure de ces reconstitutions, mais il en existe d'autres, un peu moins bonnes mais tout à fait compatibles avec l'incertitude de mesures de ces pluies moyennes sur le bassin. De plus, les pluies peuvent être spatialement fortement hétérogènes sur un massif de montagnes. Sans entrer dans les détails, on peut montrer mathématiquement qu'il est possible d'extrapoler la méthode à la reconstitution de pluies spatialement distribuées. Mais encore une fois, c'est toute une gamme de précipitations reconstituées qui peuvent être produites par la méthode. Il convient donc de contraindre ces résultats en ajoutant des informations supplémentaires pour préciser les chroniques de précipitations.

\section{3 - La météorologie}

Un réseau de pluviomètres moins dense que celui nécessaire à un échantillonnage représentatif des précipitations de montagnes pourrait compléter ce dispositif. Des informations aussi « sommaires » qu'un cumul de précipitations, qu'une valeur d'intensité pluvieuse sur une courte durée ( $3 h-6 h)$, que des informations relatives sur les pluviomètres ayant «probablement» reçu davantage de précipitations que d'autres..., sont autant d'informations qui peuvent être utilisées pour contraindre efficacement les reconstitutions de précipitations (spatialement distribuées ou non) précédemment élaborées. Ce type d'informations est souvent disponible mais actuellement inutilisable quand il s'agit de construire des chroniques de précipitations continues. Elles sont donc souvent ignorées : que faire de l'information «le seau s'est rempli au refuge du Jean Collet en 6 heures»? Toutes ces informations éparses, incomplètes, non concomitantes peuvent par contre être utilisées dans le dispositif que nous proposons et ajouter à la précision des bilans hydrométéorologiques élaborés. Elles sont moins onéreuses à construire d'un point de vue financier mais aussi et surtout, méthodologique. 


\section{Conclusion}

Depuis une vingtaine d'années, la littérature en hydrologie fait souvent état de l'impasse que constitue la critique de nos modélisations après leur calibration (Konikow et Bredehoeft, 1992). La compensation des erreurs des modèles qui sont toujours sur-paramétrés en est la cause. Cette sur-paramétrisation n'est souvent pas réductible puisqu'il faut que les modèles soient suffisamment complexes pour pouvoir répondre aux questions qui justifient leurs constructions. Mais l'insuffisance des données ne permet pas de contraindre la globalité de leurs structures.

Le manque de données hydrométéorologiques est encore plus criant dans les milieux de montagnes. La forte variabilité spatiale des variables climatiques et hydrologiques en est une cause. Les conditions souvent extrêmes que doivent supporter les capteurs installés sur le terrain en est une autre (crues/laves torrentielles, gel, faible accessibilité à l'énergie, etc.). Pour finir, les moyens financiers ne suivent pas car ils supposent d'être engagés de façon récurrente pour des résultats pas toujours faciles à valoriser aux vu des montants investis. Le besoin est pourtant grand compte tenu des nombreuses interrogations sociétales qui entourent la question des impacts des changements globaux sur les milieux de montagnes.

Nous avons proposé dans cet article une méthode permettant de vérifier la vraisemblance de la physique d'un modèle avec celle d'un bassin versant étudié. Cette méthode se fonde sur la possibilité de faire reconstituer à un modèle la chronique des précipitations étant donnée celle des débits mesurés.
Deux modèles de physiques différentes reconstituent ainsi des chroniques de précipitations significativement différentes ce qui permet de les critiquer et les discriminer en s'affranchissant des biais propres à la calibration. Cette méthode permet de retourner au paradigme perdu postulant que différentes hypothèses hydrologiques doivent conduire à des qualités de résultats discernables.

Envisagée conjointement avec l'utilisation intensive de nouveaux capteurs hydrométriques adaptés aux conditions extrêmes de l'instrumentation en montagnes (tel que le capteur HyMAGE), nous pensons qu'il est possible d'envisager cette instrumentation comme une démarche couplant l'hydrométrie et la modélisation physique, tout en soulageant la difficulté principale de constitution de réseaux de pluviomètres denses et précis.

Ceux-ci pourraient être moins performants et donc moins couteux, tout en pouvant s'installer facilement (des pluviomètres totalisateurs par exemple plutôt que des pluviomètres à haute fréquence d'acquisition). La quantité de données pluviométriques peu riches (un cumul mensuel), éparses (quelques intensités sur des périodes réduites d'un événement) ou même relatives suffisent à contraindre les résultats de l'utilisation conjointe hydrométrie-modélisation pour préciser les chroniques météorologiques.

Ces études font l'objet de tests en grandeur nature sur un torrent de montagne : le torrent du Vorz à SainteAgnès (Massif de Belledonne, Isère) et seront publiés ultérieurement.

\section{BiBLIOGRAPHIE}

Beven K.J., Kirkby M.J., 1979. A physically-based variable contributing area model of basin hydrology. Hydrological Science Bulletin, 24(1), 43-69.

Beven K.J., Lamb R., Romanowicz R., Freer J., 1995. TOPMODEL. Water Resources Publications, 627-668.

Cappus P., 1960. Etude des lois de l'écoulement. Application au calcul et à la prévision des débits. La Houille Blanche, A, 493-519.

Duband D., Obled Ch., Rodriguez J.Y., 1993. Unit hydrograph revisited: An alternate iterative approach to $\mathrm{UH}$ and effective precipitation identification. Journal of Hydrology, 150(1), 115-149.

Hewlett J., A. HibBert, 1967. Factors affecting the response of small watersheds to precipitation uin humid areas. In International Symposium on Forest Hydrology, Pergamon, New-York, 275-290.

Horton R., 1933. The role of infiltration in the hydrological cycle. Trans. American Geophysical Union, 14, 446-460 1.1.1.

KiefFer A., BoIs P., 1997. Variabilité des caractéristiques statistiques des pluies extrêmes dans les Alpes francaises. Revue des Sciences de l'Eau, 2, 199-216.
Konikow L.F., Bredehoeft J.D., 1992. Ground-water model can not be validated. Advances in water resources, 15(1), 75-83.

Le Lay M., SAulnier G.-M., 2007. Exploring the signature of climate and landscape spatial variabilities in flash flood events: Case of the 8-9 Septembre 2002 CévènnesVivarais catastrophic event. Geophysical Research Letter, 34, L13401.

Saulnier G.-M., Obled Ch., Beven K., 1998. Including spatially variable effective soil depths in TOPMODEL. Journal of Hydrology, 202, 158-172.

SAULNIER G.-M., Datin R., 2004. Analytical solving of a bias in the TOPMODEL framework water balance. Hydrological Processes, 18(7), 1195-1218.

Soll Conservation Service, 2003. Soil Conservation Service Curve Number (Scs-Cn) Methodology. Kluwer Academic Publishers, Water Science and Technology Library, $536 \mathrm{p}$. 



\section{NEIGE et GLACE de MONTAGNE \\ Reconstitution, dynamique, pratiques}

\section{Sommaire}

Editorial

Introduction

\section{1 - Reconstitution}

Coutterand S. et al. - Le lobe glaciaire lyonnais au maximum würmien : glacier du Rhône ou/et glaciers savoyards ?

Ravanel L. et al. - Désenglacement du haut bassin versant du Vorz (massif de Belledonne, Isère), au Tardiglaciaire et à l'Holocène.

Rey P.-J. - Sociétés et fluctuations du climat dans les Alpes nord-occidentales au Néolithique moyen.

Le Roy et al. - Étude des fluctuations glaciaires du Petit Âge de Glace dans le Massif des Écrins : apports de la lichénometrie.

Kirkbride M.P. - Datation des moraines holocènes d'Islande par tephrochronologie : un état de l'art.

Le Roy et al. - La dendroglaciologie, ou l'apport de l'étude des cernes d'arbres pour la reconstitution des fluctuations glaciaires holocènes.

Rabatel A. - Évolution glaciaire dans les andes subtropicales chiliennes entre 1955 et 2007 : conséquences pour la ressource en eau.

Le Roy et al. - Un inventaire des aérophotographies du massif du Mont Blanc.

\section{2 - Dynamique}

Ravanel L. - Évolution géomorphologique de la haute montagne alpine dans le contexte actuel de réchauffement climatique.

Gruber S. - Le permafrost de haute montagne.

Deline P. et al. - L'Aiguille du Midi (massif du Mont Blanc) : un site remarquable pour l'étude du permafrost des parois d'altitude.

Saulnier G.-M. et al. - Un éléphant volant est-il un oiseau ? Perspectives pour l'observation hydrométéorologique des milieux de montagne.

Jobard S. - L'instrumentation du glacier du Baounet : quels apports pour la traçabilité des mesures environnementales ?

Moreau L. - L'exploration du cryokarst glaciaire et son intérêt scientifique pour l'étude du drainage des eaux de fonte.

Mazué R. et al. - Suivi de l'évolution de la couverture detritique d'un glacier noir par photo-comparaison : le glacier d'Estelette.

\section{3 - Pratiques}

Paccard P. - Réchauffement climatique et ressource neige en domaines skiables.

Gauchon C. - Les hivers sans neige et l'économie des sports d'hiver : un phénomène récurrent, une problématique toujours renouvelée.

Laslaz L. - L'exclusion des glaciers des zones centrales des Parcs nationaux de la Vanoise et des Écrins et leur équipement pour le ski d'été.

Cayla N. - Les sentiers d'interprétation glaciaire : des outils de valorisation différenciée des glaciers et de leur territoire.

Lambert R. - Cartozonage : de la carte au zonage du risque avalanche.

Moulin A. et al. - L'incertitude liée aux avalanches dans les Alpes du Nord : identifications et implications pour la gestion.

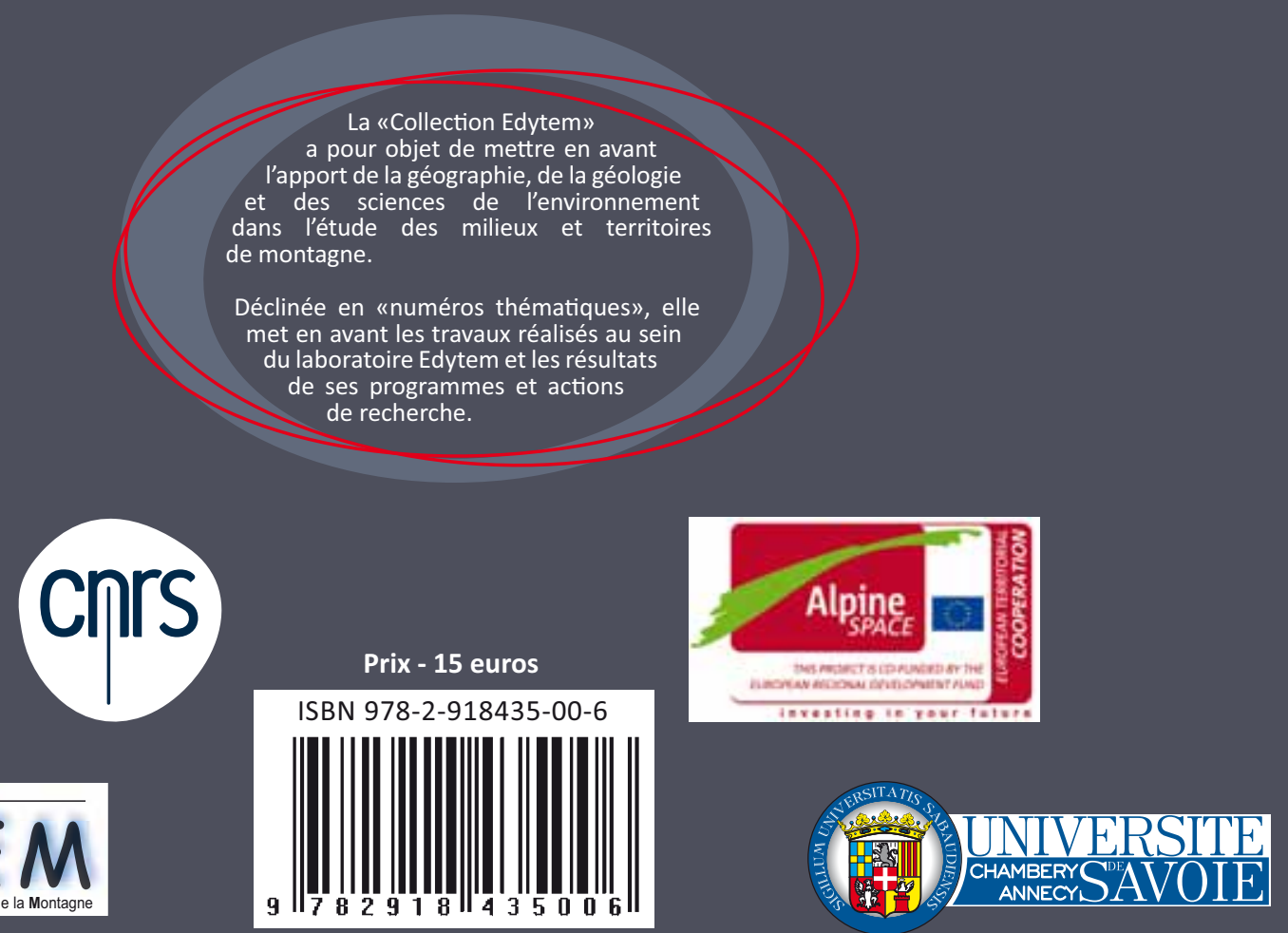

